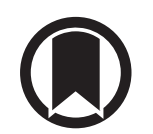

CrossMark

\title{
Riociguat for the treatment of chronic thromboembolic pulmonary hypertension: a long-term extension study (CHEST-2)
}

\author{
Gérald Simonneau ${ }^{1}$, Andrea M. D’Armini ${ }^{2}$, Hossein-Ardeschir Ghofrani ${ }^{3,4}$, \\ Friedrich Grimminger ${ }^{3}$, Marius M. Hoeper ${ }^{5}$, Pavel Jansa ${ }^{6}$, Nick H. Kim ${ }^{7}$, \\ Chen Wang ${ }^{8}$, Martin R. Wilkins ${ }^{9}$, Arno Fritsch ${ }^{10}$, Neil Davie ${ }^{10}$, Pablo Colorado ${ }^{11}$ \\ and Eckhard Mayer ${ }^{12}$
}

\begin{abstract}
Affiliations: 'Assistance Publique-Hôpitaux de Paris, Service de Pneumologie, Hôpital Bicêtre, Université Paris-Sud, Laboratoire d'Excellence en Recherche sur le Médicament et Innovation Thérapeutique, and INSERM Unité 999, Le Kremlin-Bicêtre, France. ${ }^{2}$ Division of Cardiothoracic Surgery, Foundation "I.R.C.C.S. Policlinico San Matteo", University of Pavia School of Medicine, Pavia, Italy. "University of Giessen and Marburg Lung Center (UGMLC), Giessen, Germany, and Member of the German Center of Lung Research (DZL). ${ }^{4}$ Dept of Medicine, Imperial College London, London, UK. ${ }^{5}$ Clinic for Respiratory Medicine, Hannover Medical School, Hannover, Germany, and Member of the German Center of Lung Research (DZL). ${ }^{6} \mathrm{Clinical}$ Dept of Cardiology and Angiology, First Faculty of Medicine and General Teaching Hospital, Prague, Czech Republic. ${ }^{7}$ Division of Pulmonary and Critical Care Medicine, School of Medicine, University of California San Diego, San Diego, CA, USA. ${ }^{8}$ Beijing Institute of Respiratory Medicine, Beijing Chao Yang Hospital, Dept of Respiratory Medicine, Capital Medical University, Beijing Key Laboratory of Respiratory and Pulmonary Circulation Disorders, Beijing, China. ${ }^{9}$ National Institute for Health Research/Wellcome Trust Imperial Clinical Research Facility, Imperial Centre for Translational and Experimental Medicine, Imperial College London, London, UK. ${ }^{10}$ Global Clinical Development, Bayer HealthCare Pharmaceuticals, Wuppertal, Germany. ${ }^{11}$ Global Clinical Development, Bayer HealthCare Pharmaceuticals, Barcelona, Spain. ${ }^{12}$ Kerckhoff Heart and Lung Center, Bad Nauheim, Germany.
\end{abstract}

Correspondence: Gérald Simonneau, Assistance Publique-Hôpitaux de Paris, Service de Pneumologie, Hôpital Bicêtre, Université Paris-Sud, Laboratoire d'Excellence en Recherche sur le Médicament et Innovation Thérapeutique, and INSERM Unité 999, 78 Rue du Général Leclerc, Le Kremlin-Bicêtre, 94275, France. E-mail, gerald.simonneaudabc.ap-hop-paris.fr

ABSTRACT Riociguat is a soluble guanylate cyclase stimulator approved for the treatment of inoperable and persistent/recurrent chronic thromboembolic pulmonary hypertension (CTEPH). In the 16-week CHEST-1 study, riociguat showed a favourable benefit-risk profile and improved several clinically relevant end-points in patients with CTEPH. The CHEST-2 open-label extension evaluated the long-term safety and efficacy of riociguat.

Eligible patients from CHEST-1 received riociguat individually adjusted up to a maximum dose of $2.5 \mathrm{mg}$ three times daily. The primary objective was the safety and tolerability of riociguat; exploratory efficacy endpoints included 6-min walking distance (6MWD) and World Health Organization (WHO) functional class (FC).

Overall, 237 patients entered CHEST-2 and 211 (89\%) were ongoing at this interim analysis (March 2013). The safety profile of riociguat in CHEST-2 was similar to CHEST-1, with no new safety signals. Improvements in 6MWD and WHO FC observed in CHEST-1 persisted for up to 1 year in CHEST-2. In the observed population at 1 year, mean \pm SD 6 MWD had changed by $+51 \pm 62 \mathrm{~m}(\mathrm{n}=172)$ versus CHEST- 1 baseline $(\mathrm{n}=237)$, and WHO FC had improved/stabilised/worsened in $47 / 50 / 3 \%$ of patients $(\mathrm{n}=176)$ versus CHEST-1 baseline $(\mathrm{n}=236)$.

Long-term riociguat had a favourable benefit-risk profile and apparently showed sustained benefits in exercise and functional capacity for up to 1 year.

@ERSpublications

Riociguat shows favourable benefit-risk profile in CTEPH patients, with benefits in 6MWD and WHO FC for up to 1 year http://ow.ly/Co04L 


\section{Introduction}

Chronic thromboembolic pulmonary hypertension $(\mathrm{CTEPH})$ is a form of pulmonary hypertension $(\mathrm{PH})$ caused by obstruction of the pulmonary vasculature by organised fibrotic thromboembolic material, leading to increased pulmonary vascular resistance (PVR), progressive $\mathrm{PH}$ and right ventricular failure [1-3]. Pulmonary endarterectomy (PEA) is the recommended treatment for CTEPH as it is potentially curative [4]. However, up to $40 \%$ of patients are considered technically inoperable and $17-31 \%$ of patients develop persistent or recurrent $\mathrm{PH}$ after undergoing PEA [5-8]. Until recently, no pharmacological therapies were approved for these patients.

$\mathrm{PH}$ is associated with endothelial dysfunction, impaired synthesis of nitric oxide (NO) and insufficient stimulation of the NO-soluble guanylate cyclase (sGC)-cyclic GMP (cGMP) pathway [9-11]. Riociguat, the first member of a new class of compounds called sGC stimulators, has a dual mode of action, sensitising sGC to endogenous $\mathrm{NO}$ by stabilising NO-sGC binding, and directly stimulating sGC via a different binding site, independently of NO. This leads to increased generation of cGMP [10-13].

Based on the results of the pivotal phase III CHEST-1 and PATENT-1 studies [14, 15], riociguat is the first drug to be approved for two separate $\mathrm{PH}$ indications: pulmonary arterial hypertension and inoperable or persistent/ recurrent CTEPH $[4,16]$. In the CHEST-1 study, riociguat significantly improved 6-min walking distance (6MWD) (primary end-point) with a least-squares mean difference of $+46 \mathrm{~m}$ (95\% CI 25-67 m; $\mathrm{p}<0.0001$ ) compared with placebo at week 16. A number of secondary end-points were also significantly improved compared with placebo, including PVR $(\mathrm{p}<0.0001)$, N-terminal prohormone of brain natriuretic peptide (NT-proBNP) $(\mathrm{p}<0.0001)$ and World Health Organization (WHO) functional class (FC) $(\mathrm{p}=0.003)$ [15]. In CHEST-1, there was a higher number of clinical worsening events in the placebo group versus the riociguat group ( $6 \%$ versus $2 \%$, respectively), although this was not statistically significant. Riociguat showed a favourable benefit-risk profile, with the most frequently occurring serious adverse events (SAEs) in CHEST-1 being right ventricular failure (3\% of patients in each group), syncope ( $2 \%$ in the riociguat group and $3 \%$ in the placebo group) and haemoptysis (2\% of the riociguat group) [15].

Patients completing CHEST-1 were eligible to enter the CHEST-2 long-term extension study, in which all patients received open-label riociguat, for the assessment of the long-term safety and efficacy of riociguat in patients with CTEPH. Here we report the results from an interim analysis of CHEST-2 where the majority $(76 \%)$ of patients had received $\geq 1$ year of treatment.

\section{Methods}

Patients

Patients aged 18-80 years with technically inoperable CTEPH or persistent/recurrent $\mathrm{PH}$ following PEA were included in the CHEST study. Additional inclusion and exclusion criteria have been published previously [15], and included 6MWD $150-450 \mathrm{~m}, \mathrm{PVR} \geq 300$ dyn $\cdot \mathrm{s} \cdot \mathrm{cm}^{-5}$ and mean pulmonary artery pressure $\geq 25 \mathrm{mmHg}$. Patients were invited to participate in CHEST-2 after completing CHEST-1 without ongoing study drug-related SAEs. Patients who withdrew from CHEST-1 due to clinical worsening were not allowed to participate in CHEST-2. Patients were allowed to receive endothelin receptor antagonists (ERAs) and prostanoids as add-on combination therapy during the open-label study phase of CHEST-2, if the investigator considered it to be necessary, but NO donors and specific or non-specific phosphodiesterase inhibitors were not allowed.

The study was carried out in accordance with Good Clinical Practice Guidelines and the Declaration of Helsinki. The study protocol was approved by the ethics committees of all participating centres and all patients gave their written informed consent. The CHEST-1 and CHEST-2 studies are registered at ClinicalTrials.gov (identifiers NCT00855465 and NCT00910429, respectively).

For editorial comments see Eur Respir J 2015; 45: 1211-1213 [DOI: 10.1183/09031936.00032715]

This article has supplementary material available from erj.ersjournals.com

Received: May 122014 | Accepted after revision: Sept 262014 | First published online: Nov 132014

Clinical trial: The CHEST-1 and CHEST-2 studies are registered at http://clinicaltrials.gov with identifier numbers NCT00855465 and NCT00910429, respectively.

Support statement: This study was supported by Bayer HealthCare Pharmaceuticals (Berlin, Germany). Editorial assistance was provided by Adelphi Communications Ltd (Bollington, UK), sponsored by Bayer HealthCare Pharmaceuticals.

Conflict of interest: Disclosures can be found alongside the online version of this article at erj.ersjournals.com 


\section{Study design}

CHEST-2 was a multicentre, open-label, single-group study conducted at 71 out of the 89 centres (across 25 out of the 26 countries) that participated in CHEST-1. The study consisted of an 8-week double-blind dose-adjustment phase, followed by the open-label study phase that will run until riociguat receives official approval and is commercially available. Following the approval of riociguat for the treatment of inoperable or persistent/recurrent CTEPH in the USA, Canada, Europe and Japan, some patients are now transitioning to commercially available riociguat.

Patients in CHEST-1 were randomised to receive either placebo or riociguat adjusted from a starting dose of $1 \mathrm{mg}$ three times daily up to a maximum of $2.5 \mathrm{mg}$ three times daily for 16 weeks [15]. Patients in the former riociguat group started CHEST-2 at the riociguat dose they received at the end of CHEST-1, while former placebo patients started CHEST-2 at $1 \mathrm{mg}$ three times daily. During the 8-week double-blind dose-adjustment phase of CHEST-2, patients in the former placebo group were individually adjusted up to a maximum of $2.5 \mathrm{mg}$ three times daily according to systolic blood pressure (SBP) and symptoms of hypotension, and patients in the former riociguat group continued on the dose they were receiving at the end of CHEST-1 while receiving sham titration. During the open-label study phase, investigators could adjust the riociguat dose (up to a maximum dose of $2.5 \mathrm{mg}$ three times daily) according to the patient's need, considering SBP, side-effects and progression of underlying CTEPH.

\section{Assessment}

The primary objective of the study was to assess the safety and tolerability of long-term riociguat treatment. Safety parameters included adverse events (AEs) and laboratory variables. Exploratory efficacy end-points included 6MWD, NT-proBNP, WHO FC, time to clinical worsening, Borg dyspnoea score, EuroQol 5 Dimensions (EQ-5D) questionnaire and the Living with Pulmonary Hypertension (LPH) questionnaire. Assessments took place at entry to CHEST-2, at 2, 4, 6, 8 and 12 weeks, and every 3 months thereafter. Patients who stopped study medication had a safety follow-up visit 30 days after discontinuation.

\section{Analysis}

All variables were analysed descriptively in this open-label, non-comparative study. Baseline refers to the start of CHEST-1.

At week 12 of CHEST-2, missing data were imputed according to last observation carried forward (LOCF), except in cases of death or clinical worsening without subsequent visit, where the following rules were used: 6MWD, worst possible value $(0 \mathrm{~m})$; WHO FC, worst possible score (IV) for cases of clinical worsening without subsequent visit and worst possible value plus one (V) for cases of death; Borg dyspnoea score, worst possible value (10); EQ-5D and LPH questionnaires, worst possible score. Worst possible values were not imputed for NT-proBNP.

In addition, the following exploratory missing data sensitivity analyses were performed at 1 year: LOCF, without worst case value imputation, for 6MWD; LOCF, with worst case plus one (V) imputation for cases of death, for WHO FC; and mixed model for repeated measures (MMRM) analysis for 6MWD. The MMRM was applied with baseline value, visit, former treatment group, the interaction of visit and baseline, and the interaction of visit and former treatment group as fixed effects and an unstructured covariance matrix for the observations of one patient.

Rates of survival and clinical worsening at 1 year were estimated using a Kaplan-Meier analysis, in which patients were censored if they had not reached 1 year of treatment or if they had withdrawn without experiencing an event. In addition, worst-case analyses were performed at 1 year for clinical worsening (assuming that a patient dropping out experienced clinical worsening immediately after drop-out); and survival (assuming that a patient dropping out died immediately after drop-out).

\section{Results}

\section{Patients}

Of the 243 patients who completed CHEST-1, 237 (98\%) entered CHEST-2 (fig. 1). Patient characteristics were well balanced across the groups at CHEST-1 baseline (table 1); 73\% of patients had inoperable CTEPH and 27\% had persistent/recurrent PH following PEA. At the March 2013 data cut-off, 211 patients (89\%) were receiving ongoing treatment and $179(76 \%)$ had received $>1$ year of treatment. Mean treatment duration was 83 weeks (median 75 weeks) and cumulative treatment exposure was 378 patient-years.

At the end of the 8-week double-blinded dose-adjustment phase, most patients in the former riociguat and placebo groups were receiving riociguat $2.5 \mathrm{mg}$ three times daily ( $82 \%$ and $90 \%$, respectively) (online supplementary fig. S1). This was sustained for up to 1 year, when $90 \%$ of patients were receiving riociguat 


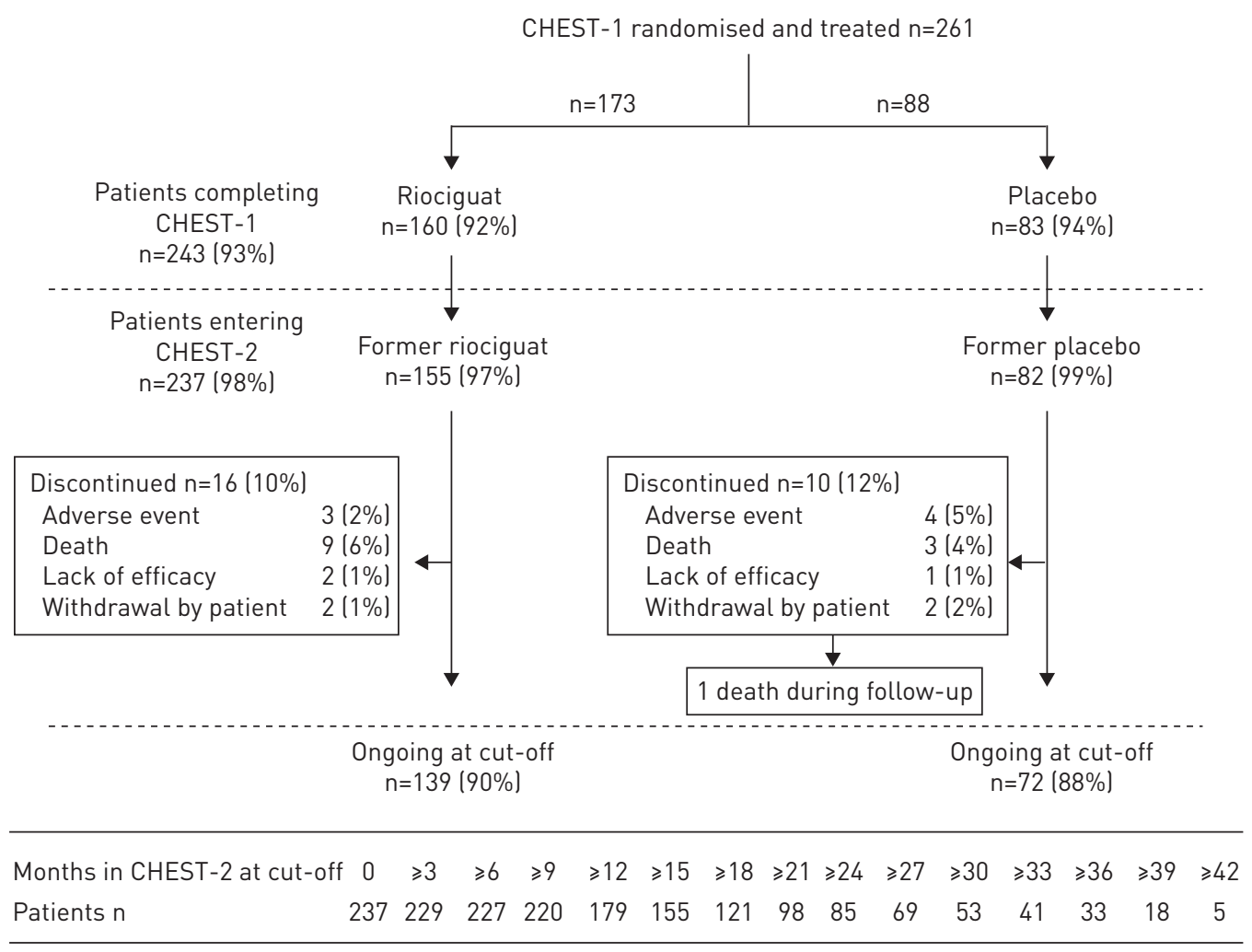

FIGURE 1 Patient disposition and numbers of patients at selected time points during the study. The numbers of patients at each time point were based on the number of visits performed.

$2.5 \mathrm{mg}$ three times daily. During the open-label study phase of CHEST-2, 23 patients received at least one up-titration of riociguat dose and 13 patients received at least one down-titration.

At the start of CHEST-2, all patients were receiving riociguat monotherapy. Of patients treated for 1 year in CHEST-2, 145 (92\%) out of 157 were continuing to receive monotherapy and 12 (8\%) patients were receiving additional $\mathrm{PH}$-specific medication (eight (5\%) were receiving ERAs and four (3\%) were receiving prostanoids). No patient required additional treatment with both an ERA and prostanoid at 1 year.

\section{Safety}

The most common AEs in CHEST-2 are shown in table 2. Hypotension was reported in 6\% of patients and syncope in $7 \%$. Compared with the initial 16-week CHEST-1 study, lower exposure-adjusted rates per 100 patient-years were seen in the long-term extension phase for the most frequent AEs and AEs of

\begin{tabular}{lccc}
\hline TABLE 1 Patient characteristics at CHEST-1 baseline & & \\
& Former riociguat & Former placebo & Total \\
\hline Subjects $\mathbf{n}$ & 155 & 82 & 237 \\
Age years & $59 \pm 14$ & $59 \pm 12$ & $59 \pm 13$ \\
Female & $104(67)$ & $49(60)$ & $153(65)$ \\
CTEPH classification & $110(71)$ & $62(76)$ & $172(73)$ \\
$\quad$ Inoperable & $45(29)$ & $20(24)$ & $65(27)$ \\
Persistent/recurrent & $345 \pm 82$ & $360 \pm 71$ & $351 \pm 78$ \\
6MWD m & $2 / 31 / 65 / 3^{\#}$ & $0 / 31 / 67 / 2^{\uparrow}$ & $1 / 31 / 65 / 3^{+}$ \\
WHO FC I/II/III/IV & & & \\
\hline
\end{tabular}

Data are presented as mean \pm SD, $n(\%)$ or $\%$, unless otherwise stated. CTEPH: chronic thromboembolic pulmonary hypertension; 6MWD: 6-min walking distance; WHO: World Health Organization; FC: functional class. ${ }^{\#}$ : data do not add up to $100 \%$ due to rounding; ${ }^{\text {ๆ}}: n=81 ;{ }^{*}: n=236$. 


\begin{tabular}{lccc}
\hline TABLE 2 Adverse events (AEs) in the CHEST-2 study & & \\
& Former riociguat & Former placebo & Total \\
\hline Subjects $\mathbf{n}$ & 155 & 82 & 237 \\
Any AE & $150(97)$ & $78(95)$ & $228(96)$ \\
Individual AEs in >10\% of patients & & & \\
$\quad$ Nasopharyngitis & $37(24)$ & $18(22)$ & $55(23)$ \\
Dizziness & $29(19)$ & $16(20)$ & $45(19)$ \\
Peripheral oedema & $24(15)$ & $19(23)$ & $43(18)$ \\
Diarrhoea & $21(14)$ & $12(15)$ & $33(14)$ \\
Cough & $20(13)$ & $12(15)$ & $32(14)$ \\
Dyspnoea & $18(12)$ & $9(11)$ & $27(11)$ \\
Upper respiratory tract infection & $18(12)$ & $8(10)$ & $26(11)$ \\
Drug-related AE & $70(45)$ & $39(48)$ & $109(46)$ \\
Discontinuation due to AE & $3(2)$ & $5(6)$ & $8(3)$ \\
Serious AEs & $63(41)$ & $37(45)$ & $100(42)$ \\
Discontinuation due to serious AE & $3(2)$ & $4(5)$ & $7(3)$ \\
AEs of special interest in >5\% of patients & $10(6)$ & $7(9)$ & $17(7)$ \\
$\quad$ Syncope & $9(6)$ & $6(7)$ & $15(6)$ \\
Hypotension & $5(3)$ & $3(4)$ & $8(3)$ \\
Other AEs of interest & & \\
Haemoptysis/pulmonary haemorrhage & & & \\
\hline
\end{tabular}

Data are presented as $\mathrm{n}(\%)$, unless otherwise stated. The mean treatment duration was 83 weeks.

special interest (online supplementary table S1). Drug-related AEs were reported in $46 \%$ of patients; the most common were dizziness (10\%), dyspepsia (8\%) and hypotension (5\%).

SAEs were reported in $42 \%$ of patients and drug-related SAEs in 5\%. The most common drug-related SAEs were syncope $(2 \%)$ and hypotension (1\%), which were resolved in all cases. Four (2\%) AEs and three (2\%) SAEs of haemoptysis/pulmonary haemorrhage were reported in the riociguat group during CHEST-1. During CHEST-2, eight (3\%) AEs and four (2\%) SAEs of haemoptysis/pulmonary haemorrhage were reported. The exposure-adjusted rate of haemoptysis/pulmonary haemorrhage AEs was 2.9 per 100 patient-years in CHEST-2 versus 7.8 per 100 patient-years in the riociguat group of CHEST-1. The exposure-adjusted rate of haemoptysis/pulmonary haemorrhage SAEs was 1.1 cases per 100 patient-years in CHEST-2 compared with 5.9 cases per 100 patient-years in the riociguat group of CHEST-1. No AEs or SAEs of haemoptysis/pulmonary haemorrhage were reported in the placebo group of CHEST-1. Two of the SAEs were graded as severe, but none were considered study drug-related by the investigators. Of the patients who experienced an SAE in CHEST-2, one had a previous history of pulmonary bleeding prior to the CHEST study (requiring bronchial artery embolisation to be carried out twice) and one had an episode of haemoptysis during CHEST-1. One $(0.4 \%)$ case was fatal and, of the remaining cases, none led to riociguat discontinuation and all were resolved or resolving at cut-off. All patients with haemoptysis/ pulmonary haemorrhage were receiving anticoagulants. Warfarin was discontinued in one patient and one patient underwent bronchial artery embolisation.

There were 13 deaths during CHEST-2, none of which were considered to be study drug-related by the investigators.

\section{Exploratory analyses}

6MWD

The reported differences in $6 \mathrm{MWD}$ relate to different numbers of patients and should therefore be considered as exploratory. Improvements in 6MWD in the riociguat group of CHEST-1 were sustained at week 12 and year 1 of CHEST-2 (fig. 2). In the former riociguat group, mean \pm SD 6MWD had changed from CHEST -1 baseline by $+50 \pm 59 \mathrm{~m}$ at week 16 of CHEST $-1(\mathrm{n}=154)$ and by $+61 \pm 59 \mathrm{~m}$ at week 12 of CHEST-2 ( $\mathrm{n}=145$; total 28 weeks). In the former placebo group, little change in 6MWD was observed at week 16 of CHEST-1 $(+8 \pm 63 \mathrm{~m}$; $\mathrm{n}=81)$; however, a change of $+51 \pm 64 \mathrm{~m}(\mathrm{n}=75)$ was observed at week 12 of CHEST-2 following transition to riociguat, which is comparable to the former riociguat group (fig. 2).

At 1 year of CHEST-2, 6MWD had changed by $+59 \pm 58 \mathrm{~m}$ for the former riociguat group $(\mathrm{n}=114),+37$ $\pm 69 \mathrm{~m}$ in the former placebo group $(\mathrm{n}=58)$ and $+51 \pm 62 \mathrm{~m}$ in the overall population $(\mathrm{n}=172)$ (fig. 2$)$. In the overall population, mean \pm SD 6 MWD was $409 \pm 96 \mathrm{~m}(\mathrm{n}=172)$ at 1 year, compared with $351 \pm 78 \mathrm{~m}$ $(\mathrm{n}=237)$ at baseline. 


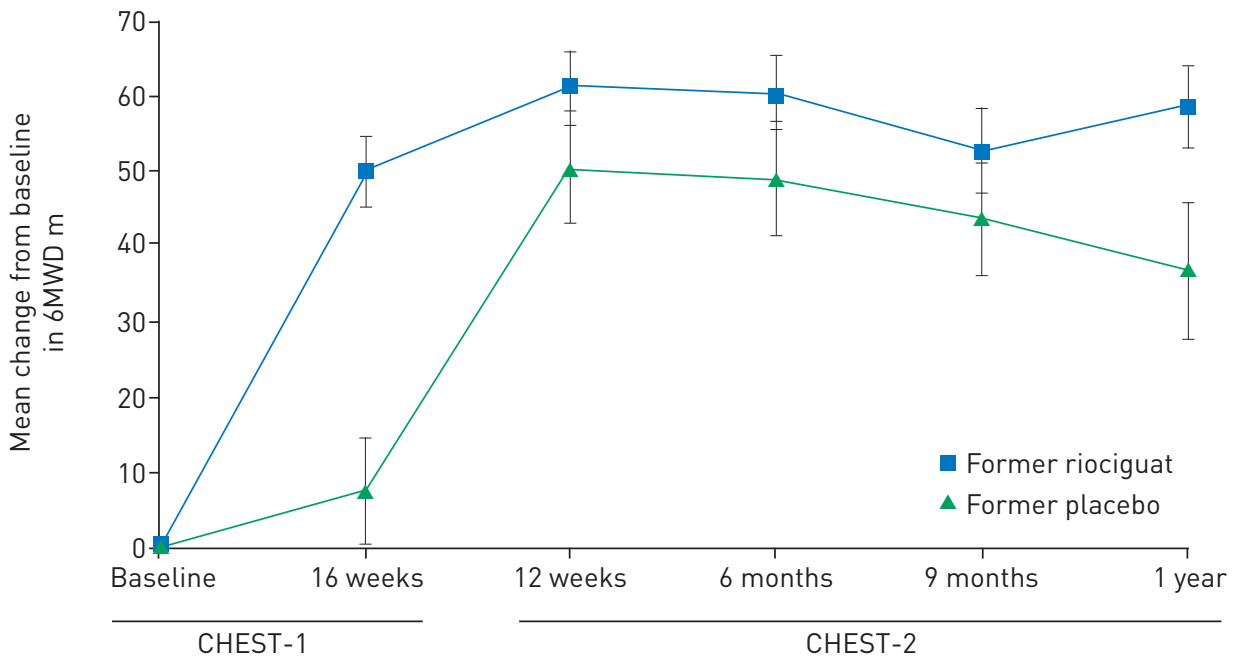

Mean 6MWD absolute values $\mathrm{m}$

Former riociguat 345

Placebo

360

Patients $\mathrm{n}$

Former riociguat 155

Placebo

82
396

368

$$
406
$$

154

81

\section{5}

75
406

411

143

75
400

411

405

114

58

FIGURE 2 Mean change from baseline in 6-min walking distance (6MWD) in CHEST-1 and CHEST-2. Data are observed values; error bars represent standard error of the mean. The reported differences in 6MWD relate to different numbers of patients at each time point and should therefore be considered exploratory.

Missing data sensitivity analyses, using different imputation methods, showed slightly lower improvements in 6MWD compared with observed values (online supplementary table S2). This was due to patients who dropped out tending to have a lower $6 \mathrm{MWD}$. However, the overall interpretation of the study results was not affected.

\section{NT-proBNP}

The decreases in NT-proBNP in the riociguat groups of CHEST-1 were sustained for up to 1 year in CHEST-2 (table 3). Patients in the placebo group of CHEST-1 showed a similar decrease in NT-proBNP following transition to riociguat in CHEST-2. At 1 year in the overall population, NT-proBNP changed by $-416 \pm 1321 \mathrm{pg} \cdot \mathrm{mL}^{-1}(\mathrm{n}=149)$ versus baseline $(\mathrm{n}=204)$.

WHO FC

At 1 year, WHO FC had improved/stabilised/worsened in 50/45/4\%, respectively, of patients in the former riociguat group $(n=117), 39 / 59 / 2 \%$ of patients in the former placebo group $(n=59)$ and $47 / 50 / 3 \%$ of

TABLE 3 Change from CHEST-1 baseline in NT-proBNP, Borg dyspnoea score, EQ-5D score and LPH score during CHEST-1 and CHEST-2

\begin{tabular}{|c|c|c|c|c|c|c|c|}
\hline & \multicolumn{3}{|c|}{ Former riociguat } & \multicolumn{3}{|c|}{ Former placebo } & \multirow{2}{*}{$\begin{array}{l}\text { Total population } \\
\text { CHEST-2 } 1 \text { year }\end{array}$} \\
\hline & CHEST-1 week 16 & CHEST-2 week 12 & CHEST-2 1 year & CHEST-1 week 16 & CHEST-2 week 12 & CHEST-2 1 year & \\
\hline $\begin{array}{l}\text { NT-proBNP pg-mL } \mathrm{mL}^{-1} \\
\text { Borg dyspnoea score } \\
\text { EQ-5D score } \\
\text { LPH score }^{+}\end{array}$ & $\begin{array}{c}-475 \pm 1428(134) \\
-1.05 \pm 2.26(154) \\
+0.10 \pm 0.23(152) \\
-9.3 \pm 16.3(152)\end{array}$ & $\begin{array}{l}-316 \pm 2350(130) \\
-1.00 \pm 2.36(145) \\
+0.13 \pm 0.24(146) \\
-13.2 \pm 16.7(138)^{\S}\end{array}$ & $\begin{array}{l}-375 \pm 1182(102) \\
-0.80 \pm 2.41(113) \\
+0.12 \pm 0.29(113)\end{array}$ & $\begin{array}{c}+146 \pm 709(68) \\
-0.06 \pm 2.15(81) \\
-0.03 \pm 0.26(79) \\
-5.0 \pm 15.0(79)\end{array}$ & $\begin{array}{c}-508 \pm 1137(65) \\
-0.70 \pm 1.93(75) \\
+0.07 \pm 0.23(75) \\
-10.8 \pm 18.9(67)^{\S}\end{array}$ & $\begin{array}{l}-505 \pm 1591(47) \\
-0.57 \pm 1.98(58) \\
+0.01 \pm 0.30(58)\end{array}$ & $\begin{array}{l}-416 \pm 1321(149) \\
-0.72 \pm 2.27(171) \\
+0.08 \pm 0.30(171)\end{array}$ \\
\hline
\end{tabular}

Data are presented as mean \pm SD (n). NT-proBNP: N-terminal prohormone of brain natriuretic peptide; EQ-5D: EuroQol 5 Dimensions; LPH: Living with Pulmonary Hypertension. $\#$ : the Borg dyspnoea scale ranges from 0 to 10 , with 0 representing no dyspnoea and 10 maximal dyspnoea; ${ }^{\text {? }}$ : Scores on the EQ-5D self-report questionnaire range from -0.6 to 1.0, with higher scores indicating a better quality of life; ${ }^{+}$: Scores on the LPH questionnaire (an adaptation of the Minnesota Living with Heart Failure Questionnaire) range from 0 to 105, with higher scores indicating worse quality of life; ${ }^{\S}$ : CHEST-2 week 8 data are shown for LPH score. 
patients in the overall population $(n=176)$ (fig. 3). The proportion of patients in the overall population in WHO FC I/II/III/IV at 1 year was $14 / 54 / 31 / 1 \%(n=177)$, respectively, compared with $1 / 31 / 65 / 3 \%(n=236)$ at baseline.

Similar to 6MWD, missing data sensitivity analyses showed slightly reduced improvements in WHO FC compared with observed values (online supplementary table 2), but the overall interpretation of study results was not affected.

\section{Survival and clinical worsening}

Overall, $16 \%$ of patients experienced a clinical worsening event during CHEST-2 (table 4). The estimated rate of clinical worsening-free survival at 1 year was $88 \%$ (95\% CI 83-92\%) (fig. 4a). Using a worst-case analysis, in which patients who dropped out were assumed to have experienced clinical worsening, the rate of clinical worsening-free survival at 1 year was $86 \%$ (95\% CI $80-90 \%)$.

The estimated overall survival rate at 1 year of CHEST-2 was 97\% (95\% CI 93-98\%) (fig. 4b). Using a worst-case analysis, where patients who dropped out were assumed to have died, the rate of survival at 1 year was $93 \%$ (95\% CI $88-96 \%)$.

\section{Quality of life}

The improvement in Borg dyspnoea score seen in the former riociguat group at the end of CHEST-1 $(n=154)$ was maintained in CHEST-2 at week $12(n=145)$ and year $1(n=113)$, while patients in the former placebo group showed minimal change at the end of CHEST-1 $(n=81)$, with improved scores after the switch to riociguat in CHEST-2 at week $12(n=75)$ and year $1(n=58)$ (table 3). Likewise, improvement in EQ-5D questionnaire score seen in the former riociguat group at the end of CHEST-1 ( $\mathrm{n}=153)$ was maintained in CHEST-2 at week $12(\mathrm{n}=147)$ and year $1(\mathrm{n}=114)$, while patients in the former placebo group showed no improvement during CHEST-1 $(n=80)$, but showed moderate improvement after the switch to riociguat in CHEST-2 at week $12(n=76)$ and year $1(n=59)$. A similar trend was observed for LPH, although this end-point was not measured beyond week 8 of CHEST-2 (table 3).

\section{Discussion}

The results of CHEST-2 support the findings from CHEST-1 and suggest that riociguat is an effective treatment for improving exercise capacity and functional capacity in patients with inoperable or persistent/ recurrent $\mathrm{PH}$. The AE profile of riociguat in CHEST-2 was similar to that seen in CHEST-1 [15], including episodes of haemoptysis. The majority of patients were receiving the maximum $2.5 \mathrm{mg}$ three times daily dose of riociguat at 1 year and changes in dose were infrequent during the open-label phase of the study, further supporting the long-term tolerability of this treatment. Furthermore, improvements in 6MWD, WHO FC and other exploratory efficacy end-points seen at the end of CHEST-1 [15] were apparently sustained for up to 1 year in CHEST-2.

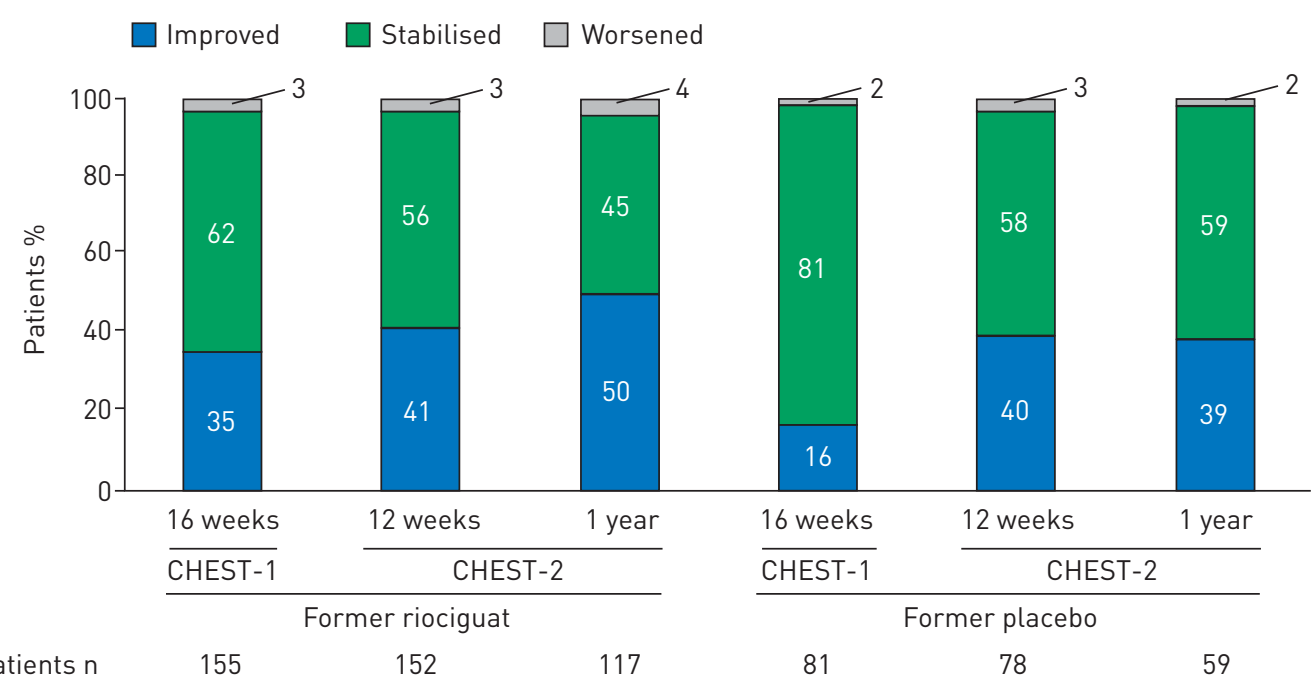

FIGURE 3 Proportion of patients in whom World Health Organization (WHO) functional class was improved/ stabilised/worsened in CHEST-1 and CHEST-2. Data are observed values. Percentages may not add up to $100 \%$ due to rounding. The reported differences in WHO functional class relate to different numbers of patients at each time point and should therefore be considered exploratory. 
TABLE 4 Clinical worsening events during CHEST-2

\begin{tabular}{lccc} 
& Former riociguat & Former placebo & Total \\
\hline Subjects n & 155 & 82 & 237 \\
Patients with clinical worsening & $26(17)$ & $12(15)$ & $38(16)$ \\
PEA & $1(1)$ & $1(1)$ & $2(1)$ \\
Hospitalisation due to PH & $5(3)$ & $1(1)$ & $6(3)$ \\
Start of new PH treatment & $12(8)$ & $7(9)$ & $19(8)$ \\
Decrease in 6MWD due to PH & $2(1)$ & $1(1)$ & $3(1)$ \\
Persistent worsening of WHO FC due to PH & $5(3)$ & $1(1)$ & $6(3)$ \\
Death & $9(6)$ & $4(5)$ & $13(5)$ \\
\hline
\end{tabular}

Data are presented as $\mathrm{n}(\%)$, unless otherwise stated. The mean treatment duration was 83 weeks. PEA: pulmonary endarterectomy; $\mathrm{PH}$ : pulmonary hypertension; 6MWD: 6-min walking distance; WHO: World Health Organization; FC: functional class. " ${ }^{\text {: }}$ one patient can experience more than one type of event.

PEA remains the recommended treatment for CTEPH as it is potentially curative in the majority of patients. Despite being an invasive procedure, the mortality rates of PEA are low: 7\% and $9 \%$ at 1 and 2 years post-surgery, respectively [7, 17]. However, there is a significant unmet medical need for effective therapies in patients who are considered technically inoperable or who develop persistent/recurrent $\mathrm{PH}$ after undergoing PEA $[5,7,8]$. Riociguat is the only approved pharmacological therapy for such patients [4] and is the first to show significant and sustained improvements in exercise capacity.

Haemoptysis and pulmonary haemorrhage are known complications of CTEPH. A previous single-centre survey found that five (6\%) out of 79 patients with CTEPH had experienced moderate-to-severe haemoptysis requiring medical intervention [18], while a recent case-control study using data from the international CTEPH registry found that $5 \%$ of 436 consecutive patients with CTEPH had a history of haemoptysis [19]. The rate of haemoptysis/pulmonary haemorrhage SAEs in CHEST-2 was 1.1 cases per 100 patient-years compared with 5.9 cases per 100 patient-years in the riociguat group of CHEST-1 (no cases were reported in the placebo group of CHEST-1). The potentially increased risk of pulmonary bleeding with riociguat in some patients is reflected as a warning in the local prescribing information (summary of product characteristics) and prescribers should regularly assess the individual patient's risk of pulmonary bleeding while on riociguat treatment. However, the risk of haemoptysis/pulmonary haemorrhage may be influenced by other factors, such as age, severity of disease, deterioration in pulmonary haemodynamics and concomitant treatment with anticoagulants $[20,21]$. The mechanism of
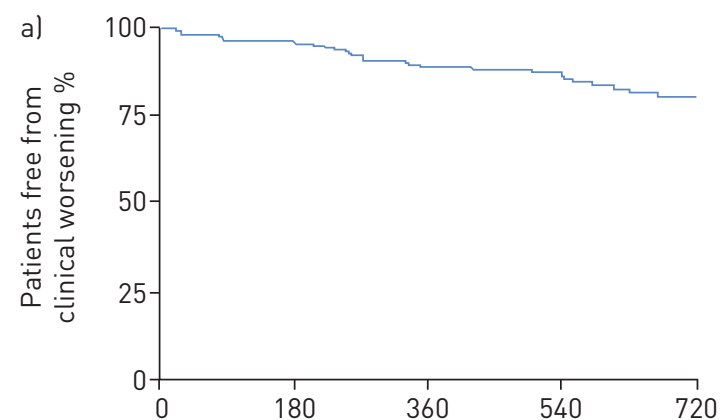

Time from start of extension study treatment days
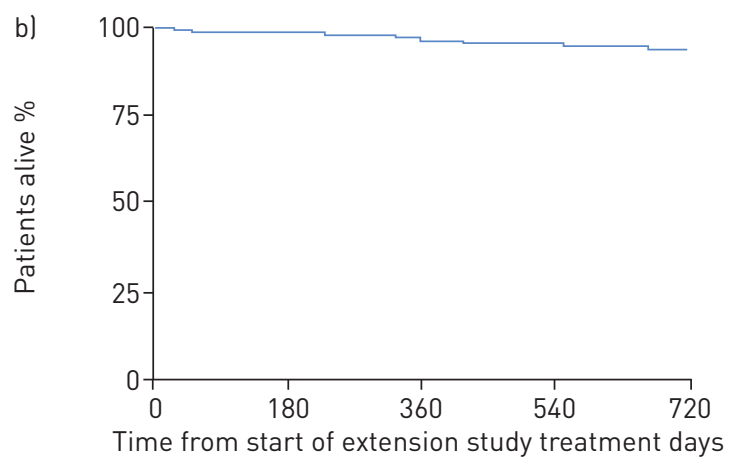

Time from start of extension study treatment days

\begin{tabular}{|c|c|c|c|c|c|c|c|c|c|c|}
\hline $\begin{array}{l}\text { Patients who reached } \\
\text { time point without } \\
\text { clinical worsening n }\end{array}$ & 237 & 218 & 155 & 96 & 65 & $\begin{array}{l}\text { Patients alive } \\
\text { at time point } n\end{array}$ & 237 & 223 & 166 & 105 \\
\hline $\begin{array}{l}\text { Patients with clinical } \\
\text { worsening } n\end{array}$ & 0 & 10 & 24 & 28 & 34 & Deaths $\mathrm{n}$ & 0 & 3 & 6 & 8 \\
\hline
\end{tabular}

FIGURE 4 Kaplan-Meier plots for a) clinical worsening and b) survival in the overall population during CHEST-2. At 1 year, the estimated rate of clinical worsening-free survival was $88 \%$ (95\% CI 83-92\%) and the estimated rate of survival was 97\% (95\% CI 93-98\%). Using a worst-case analysis for clinical worsening, where patients who dropped out were assumed to have experienced clinical worsening, the rate of clinical worsening-free survival at 1 year was $86 \%$ (95\% CI $80-90 \%)$. Using a worst-case analysis for survival, where patients who dropped out were assumed to have died, the rate of survival at 1 year was $93 \%$ (95\% CI 88-96\%). 
how riociguat could cause haemoptysis/pulmonary haemorrhage is unclear. One hypothesis is that the observation may be related to the vasodilatory effects of riociguat on the bronchial arterial circulation (which is hypertrophied and associated with angiogenesis in the systemic circulation, i.e. bronchial arteries, in $\mathrm{PH}[22,23])$, which increases the flow and favours haemoptysis.

Improvements in exercise capacity and clinical condition with riociguat during CHEST-1, as measured by 6MWD and WHO FC, were apparently sustained for up to 1 year in CHEST-2, and were consistent using a variety of imputation methods for missing data. In patients who received placebo during CHEST-1, although the incidence of clinical worsening events was similar compared with former riociguat patients, improvements in 6MWD and WHO FC after transitioning to riociguat treatment did not fully catch up to the former riociguat group by the 1-year cut-off. This highlights the importance of initiating the appropriate treatment as early as possible.

Long-term extension studies provide vital information regarding the long-term safety and tolerability of a drug; however, their lack of a control group and open-label nature in combination with patient withdrawals and the different lengths of time patients have been treated do not allow evidence-based messages regarding efficacy to be derived. In CHEST-2, patients were also allowed to receive concomitant PH medication during the open-label phase. Although the use of concomitant therapies at 1 year was low (8\%), this may have affected the $\mathrm{AE}$ profile and contributed to the improvement in efficacy parameters. Pulmonary haemodynamics were not assessed in the CHEST-2 study, and so it is unclear whether the improvements in PVR and other haemodynamic parameters persisted beyond the 16-week CHEST-1 study. However, improvements in NT-proBNP, a marker of right ventricular function, apparently persisted for up to 1 year.

In conclusion, long-term treatment with riociguat showed a favourable benefit-risk profile in patients with inoperable CTEPH or persistent/recurrent PH after PEA. As in CHEST-1, cases of haemoptysis/ pulmonary haemorrhage were observed in the CHEST-2 long-term extension. Although caution is required when interpreting open-label long-term extension studies, improvements in 6MWD and WHO FC were maintained for up to 1 year of treatment, with a survival rate of 97\% (worst-case analysis: 93\%) and a rate of clinical worsening-free survival of $88 \%$ at 1 year (worst-case analysis: $86 \%$ ). These results support the use of riociguat as a long-term treatment for patients with inoperable or persistent/recurrent CTEPH.

\section{References}

1 Lang IM, Klepetko W. Chronic thromboembolic pulmonary hypertension: an updated review. Curr Opin Cardiol 2008; 23: 555-559.

2 Peacock A, Simonneau G, Rubin L. Controversies, uncertainties and future research on the treatment of chronic thromboembolic pulmonary hypertension. Proc Am Thorac Soc 2006; 3: 608-614.

3 Simonneau G, Robbins IM, Beghetti M, et al. Updated clinical classification of pulmonary hypertension. J Am Coll Cardiol 2009; 54: Suppl., S43-S54.

4 Kim NH, Delcroix M, Jenkins DP, et al. Chronic thromboembolic pulmonary hypertension. J Am Coll Cardiol 2013; 62: Suppl., D92-D99.

5 Freed DH, Thomson BM, Berman M, et al. Survival after pulmonary thromboendarterectomy: effect of residual pulmonary hypertension. J Thorac Cardiovasc Surg 2011; 141: 383-387.

6 Lang IM. Managing chronic thromboembolic pulmonary hypertension: pharmacological treatment options. Eur Respir Rev 2009; 18: 24-28.

7 Mayer E, Jenkins D, Lindner J, et al. Surgical management and outcome of patients with chronic thromboembolic pulmonary hypertension: results from an international prospective registry. J Thorac Cardiovasc Surg 2011; 141: 702-710.

8 Wilkens $\mathrm{H}$, Lang I, Behr J, et al. Chronic thromboembolic pulmonary hypertension (CTEPH): updated Recommendations of the Cologne Consensus Conference 2011. Int J Cardiol 2011; 154: Suppl. 1, S54-S60.

9 Klinger JR. The nitric oxide/cGMP signaling pathway in pulmonary hypertension. Clin Chest Med 2007; 28: 143-167.

10 Stasch JP, Pacher P, Evgenov OV. Soluble guanylate cyclase as an emerging therapeutic target in cardiopulmonary disease. Circulation 2011; 123: 2263-2273.

11 Stasch JP, Evgenov OV. Soluble guanylate cyclase stimulators in pulmonary hypertension. Handb Exp Pharmacol 2013; 218: 279-313.

12 Follmann M, Griebenow N, Hahn MG, et al. The chemistry and biology of soluble guanylate cyclase stimulators and activators. Angew Chem Int Ed Engl 2013; 52: 9442-9462.

13 Schermuly RT, Janssen W, Weissmann N, et al. Riociguat for the treatment of pulmonary hypertension. Expert Opin Investig Drugs 2011; 20: 567-576.

14 Ghofrani HA, Galiè N, Grimminger F, et al. Riociguat for the treatment of pulmonary arterial hypertension. $N$ Engl J Med 2013; 369: 330-340.

15 Ghofrani HA, D'Armini AM, Grimminger F, et al. Riociguat for the treatment of chronic thromboembolic pulmonary hypertension. N Engl J Med 2013; 369: 319-329.

16 Galiè N, Corris PA, Frost A, et al. Updated treatment algorithm of pulmonary arterial hypertension. J Am Coll Cardiol 2013; 62: Suppl., D60-D72.

17 Simonneau G, Delcroix M, Lang IM, et al. Long-term outcome of patients with chronic thromboembolic pulmonary hypertension: results of an international prospective registry comparing operated versus non operated patients. Am J Respir Crit Care Med 2013; 187: A5365 
18 Reesink HJ, van Delden OM, Kloek JJ, et al. Embolization for hemoptysis in chronic thromboembolic pulmonary hypertension: report of two cases and a review of the literature. Cardiovasc Intervent Radiol 2007; 30: 136-139.

19 Lang IM, Simonneau G, Pepke-Zaba JW, et al. Factors associated with diagnosis and operability of chronic thromboembolic pulmonary hypertension. A case-control study. Thromb Haemost 2013; 110: 83-91.

20 Henkens IR, Hazenoot T, Boonstra A, et al. Major bleeding with vitamin $\mathrm{K}$ antagonist anticoagulants in pulmonary hypertension. Eur Respir J 2013; 41: 872-878.

21 Tio D, Leter E, Boerrigter B, et al. Risk factors for hemoptysis in idiopathic and hereditary pulmonary arterial hypertension. PLoS One 2013; 8: e78132

22 Fadel E, Wijtenburg E, Michel R, et al. Regression of the systemic vasculature to the lung after removal of pulmonary artery obstruction. Am J Respir Crit Care Med 2006; 173: 345-349.

23 Zylkowska J, Kurzyna M, Pietura R, et al. Recurrent hemoptysis: an emerging life-threatening complication in idiopathic pulmonary arterial hypertension. Chest 2011; 139: 690-693. 